\title{
The genus Testudobracon in eastern China and the description of three new species (Hymenoptera: Braconidae: Braconinae)
}

\author{
YI-PING WANG ${ }^{1,2}$, XuE-XIN CHEN $^{1, *}$ and Jun-HuA HE ${ }^{1}$ \\ ${ }^{1}$ Institute of Applied Entomology, College of Agriculture and Biotechnology, Zhejiang University, Hangzhou 310029, China \\ ${ }^{2}$ Department of Biology, College of Life Science, Zhejiang Forestry College, Lin'an 311300, China
}

Key words. Braconidae, Braconinae, Testudobracon, new species, eastern China

\begin{abstract}
The species of Testudobracon Quicke from eastern China are revised with four species recognized, including three new species: (Testudobracon flavus sp. n., Testudobracon guangxinensis sp. n. and Testudobracon grandiventris $\mathrm{sp} . \mathrm{n}$.). The new species are fully described and illustrated. A key to species of this genus is also provided. The types and other specimens are deposited in the Collection of Parasitic Hymenoptera at the Zhejiang University, Hangzhou, China.
\end{abstract}

\section{INTRODUCTION}

The genus Testudobracon Quicke, 1986 of the subfamily Braconinae belonging to family Braconidae (Hymenoptera) was erected with Testudobracon niger Quicke, 1986 as its type species (Quicke, 1986). This small genus included six species before this study, mainly distributed in the Afrotropical (undescribed species) and Indo-Australian regions (Quicke \& Ingram, 1993). The species of Testudobracon are apparently specialist ectoparasitoids of gall-forming cecidomyiid Diptera, especially and perhaps exclusively the Asphonolylini (Quicke, 1986; Maetô, 1991).

The species of the genus Testudobracon in China are revised in this paper, and four species are reported. Among them, three species are identified as new to science, i.e., T. flavus sp. n., T. guangxinensis sp. n. and $T$. grandiventris $\mathrm{sp} . \mathrm{n}$. The types and other specimens are deposited in the Collection of Parasitic Hymenoptera at Zhejiang University, Hangzhou, China.

For the identification of this genus see Quicke (1987), and for the morphological terminology used in this paper see Achterberg (1979) and Quicke (1987).

\section{KEY TO SPECIES OF THE GENUS TESTUDOBRACON}

1 Median emargination of 6th metasomal tergite shallow, its maximum depth far less than 0.3 times the median length of 6th metasomal tergite, and not touching the basal sulcus of 6th metasomal tergite (Figs 32, 34); ovipositor sheath at least 0.75 times length of fore wing $\ldots \ldots \ldots \ldots \ldots 2$

- Median emargination of 6th metasomal tergite deep, its maximum depth more than 0.4 times the median length of 6th metasomal tergite (Figs 8, 16, 18, 26, 28), sometimes touching the basal sulcus of 6 th metasomal tergite (Figs 9, 35 ); ovipositor sheath less than 0.75 times length of fore

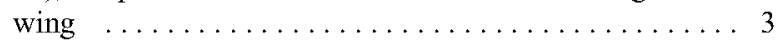

2 Hind legs almost entirely pale yellowish brown; 4th-6th metasomal tergites largely whitish, except for yellowish basal (anterior) third and postero-lateral protuberances somewhat darker; vertex with distinct fine transverse striation; 4th-6th metasomal tergites foveately rugulose (Fig. 34). Japan . . . . . . . . . T. longicaudis Maetô, 1991 - Hind legs almost entirely black; 4th-6th metasomal tergites with basal (anterior) half black or piceous brown, and posterior half white; vertex totally smooth and shiny; 4th-6th metasomal tergites irregularly punctate and weakly sculptured (Fig. 32). Australia . . . . . . . . . ..........

T. australicolorus Quicke \& Ingram, 1993

3 Length of body less than $2.5 \mathrm{~mm}$; metasoma more elongate; postero-lateral protuberances of 6 th metasomal tergite barely visible in dorsal view (Figs 8,9) $\ldots \ldots \ldots 4$

- Length of body more than $2.6 \mathrm{~mm}$; metasoma less elongate; postero-lateral protuberances of 6 th metasomal tergite distinctly visible in dorsal view (Figs 16, 18, 26, 28, 35) . . 5

4 Median emargination of 6th metasomal tergite not touching the basal sulcus of 6 th metasomal tergite, and its maximum depth about 0.5 times median length of 6 th metasomal tergite (Fig. 8); 5th metasomal tergites with strongly rounded postero-lateral lobe-like protuberances in lateral view (Fig. 7); body and legs uniformly yellow. Eastern China ...... T. flavus sp. $\mathrm{n}$.

- Median emargination of 6th metasomal tergite touching the basal sulcus of 6th metasomal tergite (Fig. 9), 5th metasomal tergites with weak postero-lateral protuberances in lateral view; body and legs pale brownish yellow except for black antenna and ovipositor sheath. Australia .........

T. tatyanae Quicke \& Ingram, 1993

5 Postero-lateral protuberances of 6 th metasomal tergite acute in lateral view (Figs 27, 29,36) $\ldots \ldots \ldots \ldots \ldots \ldots 6$

- Postero-lateral protuberances of 6th metasomal tergite rounded, lobe-like in lateral view (Fig. 17) ....... 8

6 Median emargination of 6 th metasomal tergite touching the basal sulcus of 6th metasomal tergite (Fig. 35), 4th-5th metasomal tergites with large and rounded postero-lateral, lobe-like protuberances in lateral view (Fig. 36); body and legs entirely brownish yellow. Australia ............ T. unicolorus Quicke \& Ingram, 1993

\footnotetext{
* Corresponding author. E-mail: xxchen@zju.edu.cn
} 
- Median emargination of 6th metasomal tergite not touching the basal sulcus of 6th metasomal tergite (Figs 26, 28), 4th-5th metasomal tergites with small and weak rounded postero-lateral protuberances in lateral view (Figs 27, 29); body and legs largely brown or blackish-brown ..... 7

7 Depth of median emargination of 6th metasomal tergite more than 0.4 times the median length of 6 th metasomal tergite (Fig. 28); middle lobe of mesoscutum largely setose (Fig. 31); length of vein 1-SR about 0.3 times that of fore wing vein 1-M (Fig. 30); median area of 2nd-3rd metasomal tergites pale brown; width of 3rd metasomal tergite less than 3.1 times that of median length. Eastern China, Japan .....

T. pleuralis (Ashmead, 1906)

Depth of median emargination of 6 th metasomal tergite less than 0.4 times the median length of 6 th metasomal tergite (Fig. 26); middle lobe of mesoscutum largely glabrous (Fig. 22 ); length of vein 1 -SR about 0.4 times that of fore wing vein 1-M (Fig. 24); median area of 2 nd-3rd metasomal tergites blackish brown (Fig. 25); 3rd metasomal tergite at least 3.3 times as wide as its median length (Fig. 25). Eastern China $\ldots \ldots \ldots \ldots \ldots \ldots$ T. grandiventris $\mathrm{sp} . \mathrm{n}$

8 Second metasomal tergite with a pair of sub-parallel sublateral grooves (Fig. 15); vein $1-\mathrm{SR}+\mathrm{M}$ of fore wing straight, and vein 1 -SR of forewing 0.3 times as long as vein 1-M (Fig. 14); depth of median emargination of 6th metasomal tergite 0.75 times its median length (Fig. 16); body largely brown. Eastern China

T. guangxinensis sp. $\mathrm{n}$

- Second metasomal tergite without a pair of sub-parallel sublateral grooves; vein $1-S R+M$ of fore wing slightly curved basally towards posterior margin, and vein 1-SR of fore wing 0.4 times as long as vein 1-M (Fig. 19); depth of median emargination of 6th metasomal tergite 0.60 times its median length (Fig. 18); body largely black. Java ....... .

T. niger Quicke, 1986

\section{Testudobracon flavus sp. $\mathbf{n}$.}

(Figs 1-8)

Diagnosis. This new species is closely related to $T$. tatyanae but differs from the latter by having median emargination of 6 th metasomal tergite not touching the basal sulcus of 6th tergite, and about 0.5 times the length of 6th metasomal tergite medially (Fig. 8); body and legs uniformly yellow; 2nd metasomal tergite with sub-parallel sub-lateral grooves; 2nd-3rd metasomal tergites with sparse and shallow foveate rugosity (Fig. 6); 3rd-5th metasomal tergites with distinctly rounded postero-lateral, lobe-like protuberances in lateral view, and 6th metasomal tergite with small acute postero-lateral protuberances in lateral view (Fig. 7); and length of ovipositor sheath 0.65 times that of fore wing.

\section{Description}

Female. Length of body $2.45 \mathrm{~mm}$, of fore wing 2.65 $\mathrm{mm}$ and of ovipositor sheath $1.85 \mathrm{~mm}$.

Head (Figs 1, 2). Antennal segments 24-25, length of first flagellomere 1.2 times that of second, length of first, second and penultimate flagellomeres $2.4,2.0$ and 1.9 times their maximum width, respectively. Face with short setae, and distinctly medio-longitudinal ridge (Fig. 1); face weakly convex. Eyes glabrous slightly emarginated, height of eye : width of face : width of head $=7: 8: 17$ (Fig. 1). Shortest distance between eyes 0.54 times width of head. Length of eyes 2.2 times temple in dorsal view; temple sparsely punctate. Height of clypeus : intertentorial distance : tentorio-ocular distance $=11: 24: 18$. Vertex slightly convex, coriaceous and finely setose. Frons coriaceous and finely setose. Clypeus with weak dorsal carina; malar suture distinct, length of malar space 1.1 times basal width of mandible (Fig. 1). Shortest distance between posterior ocellus : transverse diameter posterior ocellus : shortest distance between posterior ocellus and eye $=17: 11: 32$. (Fig. 2). Horizontal length of eye 2.5 times that of temple behind eye (Fig. 2).

Mesosoma. Mesosoma approximately as long as high. Mesosoma smooth and shiny (Fig. 3). Pronotum partially setose. Mesoscutum finely punctate. Middle lobe of mesoscutum flat or somewhat concave anteriorly in dorsal view. Notauli deeply impressed and smooth, and rounded posteriorly, not touching scutellar sulcus. Mesoscutal lobes evenly setose. Scutellum with sparse and short setae (Fig. 3); metanotum with a mediolongitudinal carina (Fig. 4). Propodeum smooth and shiny with short setae laterally, its medio-longitudinal ridge weak, posteriorly diverging into two weak branches (Fig. 4).

Wings. Fore wing (Fig. 5). Pterostigma 2.8 times longer than maximum width; vein $\mathbf{r}$ arising half way along pterostigma. Length of vein 1-SR 0.4 times that of vein 1-M. Ratio of length of veins $2-\mathrm{SR}+\mathrm{M}: 2-\mathrm{M}: \mathrm{m}-\mathrm{cu}$ $=6: 23: 5$. Ratio of length of veins 2 -CU1 $: 3-\mathrm{CU} 1=13$ : 5. Vein 2-SR slightly sinuate; ratio of length of veins SR1 $: 3-S R: r=63: 20: 9$. Ratio of length of veins $2-S R$ $: 3-\mathrm{SR}: \mathrm{r}-\mathrm{m}=13: 16: 7$. Hind wing without a single bristle at the apex of vein $\mathrm{C}+\mathrm{SC}+\mathrm{R}$. Ratio of length of veins $1 \mathrm{r}-\mathrm{m}: \mathrm{SC}+\mathrm{R} 1=1: 2$. Length of vein $2-\mathrm{SC}+\mathrm{R} 0.2$ times that of vein $1 \mathrm{r}-\mathrm{m}$.

Legs. Ratio of length of fore femur : tibia : tarsus $=11$ : $13: 9$. Length of fore femur and tibia 4.0 and 6.8 times their maximum depth, respectively. Hind coxa sparsely setose, virtually finely punctate, nearly smooth. Ratio of length of hind femur : tibia : basitarsus $=13: 16: 5$. Length of hind femur and tibia 3.8 and 7.2 times their maximum depth. Length of hind basitarsus 5.1 times its maximum depth.

Metasoma. Tergites with sparse and short setae. Metasoma more elongate than in the other species. Length of first metasomal tergite 0.7 times its apical width. First tergite with a posterior carina area (Fig. 6). 2nd-3rd metasomal tergites 2.5 times and 2.4 times as wide as their maximum median length, respectively, with uniformly foveate rugae and a pair of sub-parallel sub-lateral grooves medially on second metasomal tergite (Fig. 6); 2nd-3rd tergites combined about 0.6 times length of metasoma. 4th-6th metasomal tergites with weakly foveate rugae; 4th-5th metasomal tergites with postero-lateral rounded protuberances in lateral view (Fig. 7). 6th metasomal tergite with small postero-lateral angular protuberances in lateral view (Fig. 7), and a deep semicircular median emargination, but not touching the basal sulcus of 6 th metasomal tergite, and about 0.5 times the maximum length of 6 th tergite medially (Fig. 8). Length of ovi- 

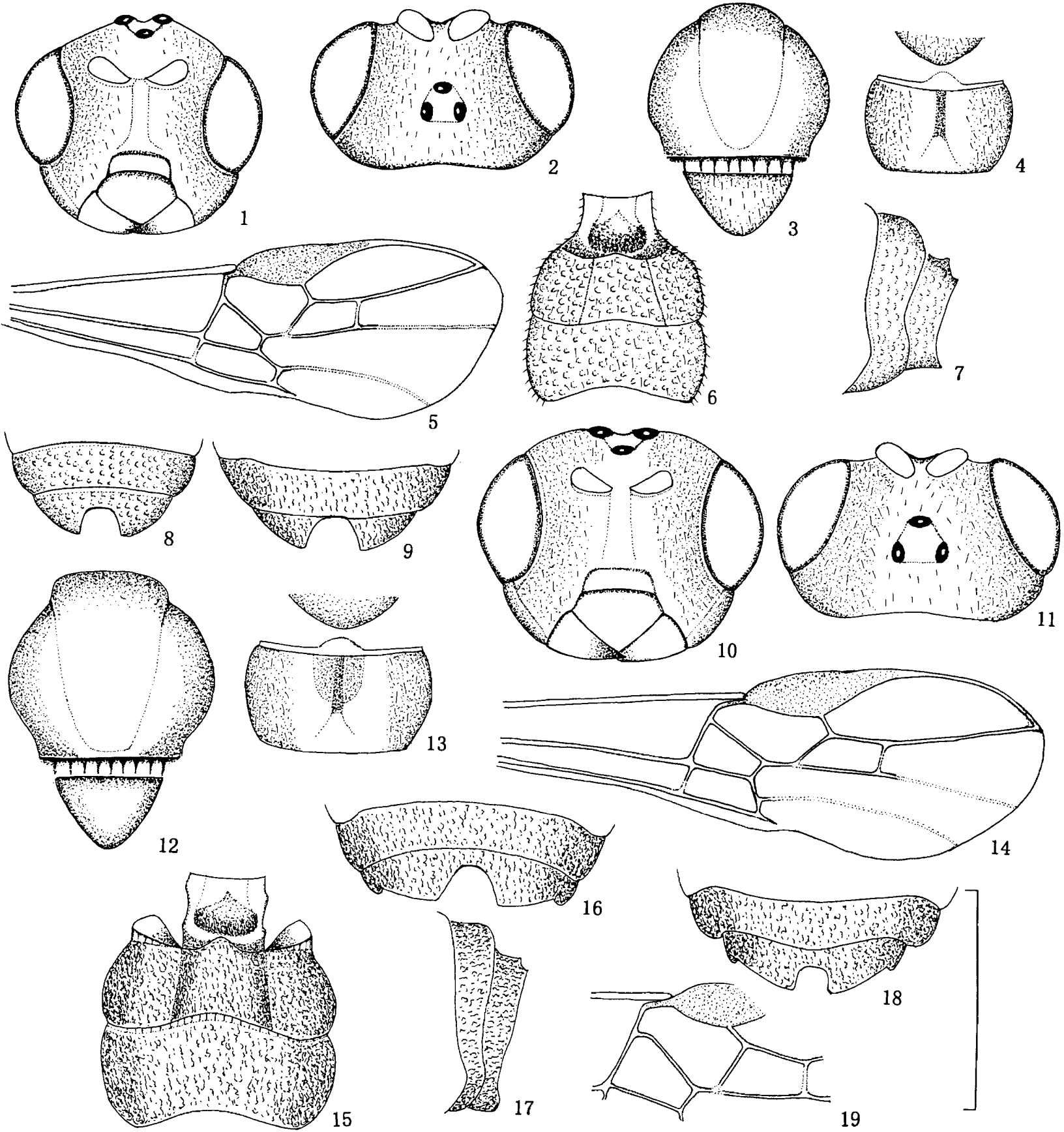

Figs 1-19. 1-8 - Testudobracon flavus sp. n.; 9 - Testudobracon tatyanae Quicke \& Ingram (Redrawn after Quicke \& Ingram, 1993); 10-17 - Testudobracon guangxinensis sp. n; 18-19 - Testudobracon niger Quicke (Redrawn after Quicke, 1986). 1, 10 head, frontal view; 2,11 - head, dorsal view; 3, 12 - mesoscutum, dorsal view; 4, 13 - propodeum; 5, 14, 19 - fore wing; 6 , 15 1st-3rd metasomal tergites, dorsal view; 7, 17 - 5th-6th metasomal tergites, lateral view; 8, 9, 16, 18 - 5th-6th metasomal tergites, dorsal view. Scale bar $=0.83 \mathrm{~mm}$ (Figs 1-3, 10-12); $0.63 \mathrm{~mm}$ (Figs 4, 13); $1.05 \mathrm{~mm}$ (Figs 5, 14, 19); $1.10 \mathrm{~mm}($ Figs 6, 15); $0.82 \mathrm{~mm}$ (Figs 7, 17); $1.20 \mathrm{~mm}$ (Figs 8, 9, 16, 18).

positor 1.2 times that of metasoma, and about 0.7 times as long as forewing. Setosity of ovipositor sheaths sparse and long.

Body entirely uniformly pale yellow or yellow except for dark brown antennae, veins, and ovipositor sheath, and black pterostigma and telotarsus.

Type material. Holotype, $q$ (China): "Zhejiang, Hangzhou, v.1975, He Jun-hua". Paratypes: 1 \&(China): "Zhejiang, Hang- zhou, 6.v.1979, Wang Hui-ping"; 1 क (China): "Jiangsu, Nanjing, 1995, Huang Qi-lin".

Etymology. From "flavus", Latin for yellow, referring to the body colour.

Distribution. China (Jiangsu, Zhejiang).

\section{Testudobracon guangxinensis sp. $\mathbf{n}$.}

(Figs 10-17)

Diagnosis. This new species is closely related to $T$. niger but differs from the latter by having body largely 

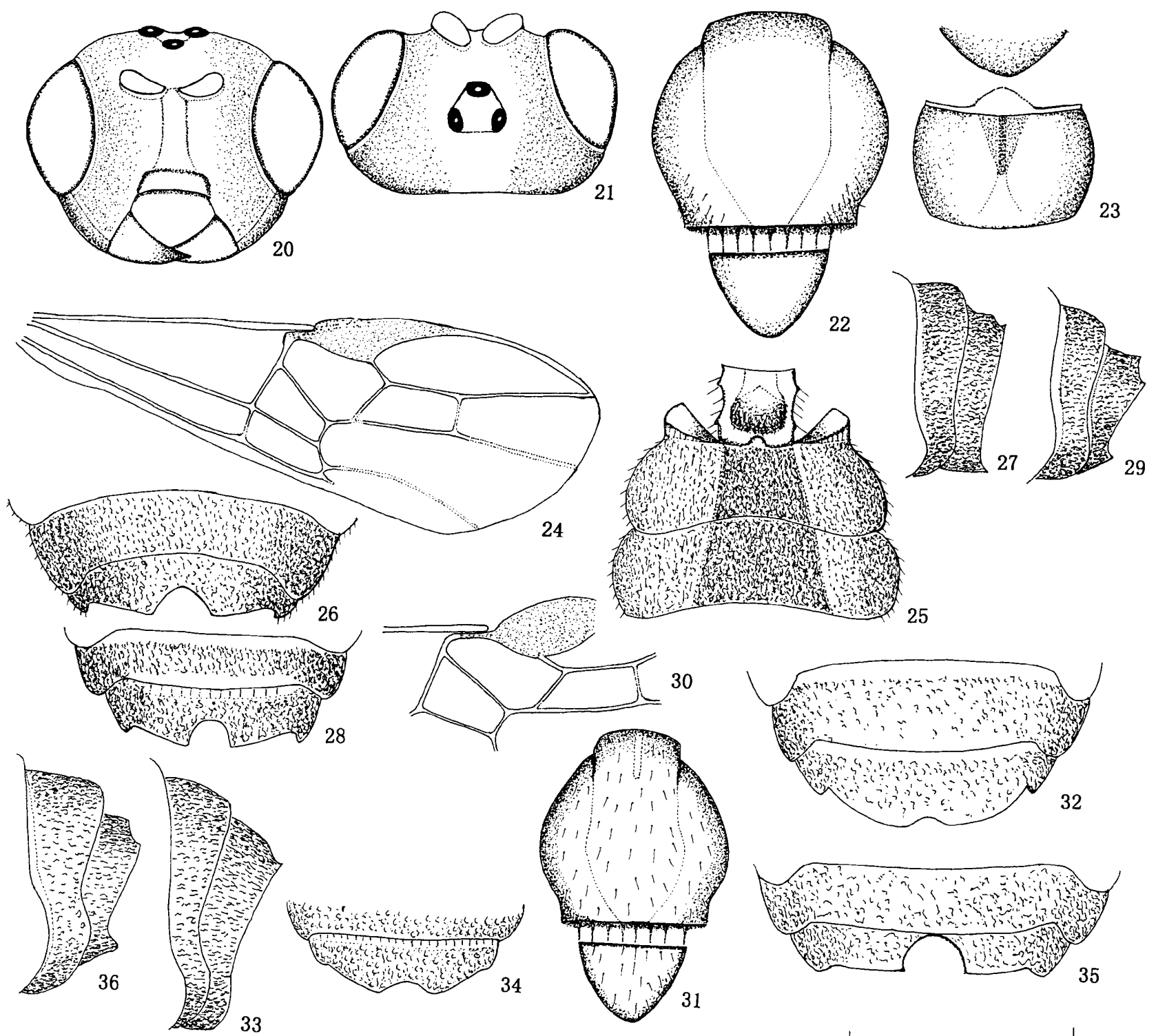

Figs 20-36. 20-27 - Testudobracon grandiventris sp. n.; 28-31 - Testudobracon pleuralis Quicke; 32-33 - Testudobracon australicolorus Quicke \& Ingram (Redrawn after Quicke \& Ingram, 1993); 34 - Testudobracon longicaudis Maetô (Redrawn after Maetô, 1991); 35-36 - Testudobracon unicolorus Quicke \& Ingram (Redrawn after Quicke \& Ingram, 1993). 20 - head, frontal view; 21 - head, dorsal view; 22, 31 - mesoscutum, dorsal view; 23 - propodeum; 24, 30 - fore wing; 25 - 1st-3rd metasomal tergites, dorsal view; 26, 28, 32, 34, 35 - 5th-6th metasomal tergites, dorsal view; 27, 29, 33, 36 - 5th-6th metasomal tergites, lateral view. Scale bar $=0.83 \mathrm{~mm}$ (Figs 20-22, 31); $0.63 \mathrm{~mm}$ (Fig. 23); $1.05 \mathrm{~mm}$ (Figs 24, 30); $1.10 \mathrm{~mm}$ (Fig. 25); 1.20 mm (Figs 26, 28, 30, 32, 34, 35); $0.82 \mathrm{~mm}$ (Figs 27, 29, 33, 36).

brown; fore wing vein $1-\mathrm{SR}+\mathrm{M}$ straight, and the length of vein 1-SR 0.3 times vein 1-M (Fig. 14); notauli weak but distinct, not touching scutellar sulcus posteriorly (Fig. 12); scutellar smooth and shiny, without setae (Fig. 12); propodeum with depressed pit antero-medially (Fig. 13); 2nd metasomal tergite with a pair of sub-parallel sublateral grooves (Fig. 15); depth of median emargination of 6 th metasomal tergite 0.75 times the length of 6 th tergite medially (Fig. 16).

\section{Description}

Female. Length of body $3.10 \mathrm{~mm}$, of fore wing 3.15 $\mathrm{mm}$ and length of ovipositor sheath $2.25 \mathrm{~mm}$.

Head (Figs 10, 11). Antennal segments 25 or 26, length of first flagellomere 1.2 times that of second, length of first, second and penultimate flagellomeres 2.2, 1.8 and 2.0 times their maximum width, respectively. Face weakly punctate and shortly setose, and with wide mediolongitudinal carina (Fig. 10). Length of face 0.7 times width. Eyes glabrous and not emarginated, height of eye : width of face : width of head $=9: 11: 20$ (Fig. 10). Shortest distance between eyes 0.58 times width of head. Length of eye 2.0 times temple in dorsal view. Vertex and face sparsely minutely punctate and densely setose. Height of clypeus : inter-tentorial distance : tentorioocular distance $=11: 25: 19$. Frons coriaceous and shortly setose. Shortest distance between posterior ocellus : transverse diameter posterior ocellus : shortest distance between posterior ocellus and eye $=15: 10: 31$ (Fig. 11). 
Horizontal length of eye 2.6 times that of temple behind eye (Fig. 11).

Mesosoma. Length of mesosoma slightly longer than its height. Middle lobe of mesoscutum flat and glabrous, without medio-longitudinal ridge. Lateral lobe of mesoscutum finely punctate, nearly smooth. Notauli weak but distinct, not touching scutellar sulcus posteriorly (Fig. 12). Scutellum glabrous. Mesopleura with dense and long setae antero-medially. Propodeum densely granulate, with distinct medio-longitudinal ridge and depressed anteriormedian pit, laterally with short silvery hairs (Fig. 13).

Wings. Fore wing (Fig. 14). Length of pterostigma 3.1 times its width; vein $r$ arising less than half way $(0.4)$ along the pterostigma. Vein $1-\mathrm{SR}+\mathrm{M}$ straight. Length of vein 1-SR 0.3 times that of vein $1-\mathrm{M}$. Ratio of length of veins $2-\mathrm{SR}+\mathrm{M}: 2-\mathrm{M}: \mathrm{m}-\mathrm{cu}=7: 22: 4$. Ratio of length of veins 2 -CU1 $: 3$-CU1 $=11: 4$. Ratio of length of veins SR : 3-SR : $r=65: 23: 10$. Ratio of length of veins 2-SR : 3-SR : $\mathrm{r}-\mathrm{m}=14: 18: 9$. Hind wing without a single bristle at the apex of vein $\mathrm{C}+\mathrm{SC}+\mathrm{R}$; ratio of length of veins $1 \mathrm{r}-\mathrm{m}: \mathrm{SC}+\mathrm{R} 1=11: 16$

Legs. Ratio of length of fore femur : tibia : tarsus $=11$ : $14: 10$; length of fore femur 4.3 and 6.7 times their maximum depth. Hind coxa smooth, hind tibia with shallow latero-longitudinal depression. Ratio of length of hind femur : tibia : basitarsus $=17: 20: 6$. Length of hind femur and tibia 3.8 and 7.0 times their maximum depth, respectively. Length of hind basitarsus 5.1 times its maximum depth.

Metasoma. First metasomal tergite 0.9 times as long as its apical width. Maximum width of 2 nd-3rd metasomal tergites 2.9 times and 3.0 times their median length, respectively. 2 nd-3rd metasomal tergites combined about 0.65 times length of metasoma, and with distinct sculpture. 2nd metasomal tergite with a pair of sub-parallel, sub-lateral, shallow grooves (Fig. 15). 4th-5th metasomal tergites with weakly rounded postero-lateral protuberances (Fig. 17). 6th metasomal tergite with large posterolateral, lobe-like protuberances in lateral view (Fig. 17), and a deep, wide median emargination in dorsal view. The maximum depth of emargination about 0.75 times the length of 6th tergite medially (Fig. 16). Length of ovipositor 1.1 times length of metasoma, and about 0.6 times as long as fore wing. Setosity of ovipositor sheaths sparse and evenly short.

Head brownish-yellow, antenna dark brown, palpi and legs pale yellow; mesosoma reddish brown; metasoma largely reddish brown except for third tergite blackish brown baso-medially; pterostigma brownish-yellow.

Type material. Holotype, $q$ (China): "Guangxi, Guanyang, 14.x.1981, Deng Gen-ming". Paratype, क(China): "Guangxi, Longsheng, 12.ix.1980, Zhou Zhi-hong".

Etymology. From "Guangxi", referring to the type locality. Distribution. China (Guangxi)

\section{Testudobracon grandiventris sp. $\mathbf{n}$.}

(Figs 20-27)

Diagnosis. This species is closely related to $T$. pleuralis, but can be separated from the latter as the middle lobe of mesoscutum is mainly glabrous; length of vein $1-\mathrm{SR}$ of fore wing about 0.4 times vein $1-\mathrm{M}$ (Fig. 24); 2nd-3rd metasomal tergites dark brown medially (Fig. 25); the metasomal tergites relatively larger and wider, and 3 rd metasomal tergite at least 3.3 times as wide as its median length (Fig. 25); propodeum with slightly depressed triangular pit medio-anteriorly (Fig. 23); maximum depth of median emargination of 6th metasomal tergite about 0.4 times the length of 6 th tergite medially (Fig. 26).

\section{Description}

Female. Length of body $3.20 \mathrm{~mm}$, of fore wing 3.35 $\mathrm{mm}$ and of ovipositor sheath $2.40 \mathrm{~mm}$.

Head (Figs 20, 21). Antenna with 24-25 segments, length of first flagellomere 1.2 times that of second, length of first, second and penultimate flagellomeres 2.3, 2.0 and 1.8 times their maximum width, respectively. Face smooth and shiny medially, coriaceous and finely punctate laterally, and with raised area (ridge) mediolongitudinally (Fig. 20). Eyes glabrous and not distinctly emarginated, height of eye : width of face : width of head $=9: 11: 20$. Length of face 0.45 times its width (Fig. 20). Shortest distance between eyes 0.53 times width of head. Temple smooth, roundly narrowed posteriorly. Vertex and face smooth and shiny, and only sparsely minutely punctate. Height of clypeus : intertentorial distance : tentorio-ocular distance $=11: 24: 20$ (Fig. 20). Malar space 0.24 times height of eyes. Shortest distance between posterior ocellus : transverse diameter posterior ocellus : shortest distance between posterior ocellus and eyes $=18: 11: 33$. Horizontal length of eyes 2.7 times that of temple behind eyes (Fig. 21).

Mesosoma. Length of mesosoma 1.2 times its height. Mesoscutum smooth and shiny; a pair of notauli meeting scutellar sulcus posteriorly (Fig. 22); middle lobe of mesoscutum glabrous, and densely setose posterolaterally (Fig. 22). Mesopleuron smooth, without setae. Metanotum with raised medio-longitudinal area, and long setae laterally. Propodeum smooth and shiny, densely finely punctate laterally, and with slightly depressed triangular pit antero-medially, and without setae laterally (Fig. 23).

Wings. Fore wing (Fig. 24). Pterostigma 3.4 times longer than its maximum width; vein $r$ arising slightly less than half way $(0.45)$ along the pterostigma. Length of vein 1-SR 0.4 times vein $1-\mathrm{M}$. Ratio of length of veins $2-\mathrm{SR}+\mathrm{M}: 2-\mathrm{M}: \mathrm{m}-\mathrm{cu}=6: 26: 5$. Ratio of length of veins 2 -CU1 : 3-CU1 = $21: 6$. Ratio of length of veins SR1 : 3-SR : $r=59: 26: 10$. Vein $1-S R+M$ virtually straight. Ratio of length of veins $2-\mathrm{SR}: 3-\mathrm{SR}: \mathrm{r}-\mathrm{m}=12$ : $17: 9$. Hind wing with a single bristle at the apex of vein $\mathrm{C}+\mathrm{SC}+\mathrm{R}$. Ratio of 1ength of $1 \mathrm{r}-\mathrm{m}: \mathrm{SC}+\mathrm{R} 1=5: 19$.

Legs. Ratio of length of fore femur : tibia : tarsus $=10$ : $11: 8$. Length of fore femur and tibia 4.1 and 6.5 times their maximum width, respectively. Hind coxa smooth and densely setose. Ratio of length of hind femur : tibia : basitarsus $=10: 13: 4$. Length of hind femur and tibia 3.6 and 6.9 times their maximum depth. Length of basitarsus 5.3 times its maximum depth. 
Metasoma. Metasoma distinctly larger and wider than that of the others species, and with dense fine setae. Length of first metasomal tergite 0.9 times its apical width, with setae on lateral margin (Fig. 25). 2nd metasomal tergite 3.1 times as wide as its medial length; 2nd-3rd metasomal tergites coarsely areolate-rugose, with a pair of sub-parallel sub-lateral grooves. 3rd metasomal tergite 3.3 times as wide as its medial length (Fig. 25); 2nd-3rd metasomal tergites combined about 0.67 times length of metasoma. 4th-5th tergites with rounded lobelike postero-lateral protuberances in lateral view (Fig. 27). Posterior margin of 6th tergite with a deep and distinct median emargination, and the maximum depth about 0.4 times the length of 6th tergite medially (Fig. 26). 6th metasomal tergite with sharp angular postero-lateral protuberances in lateral view (Fig. 27). Length of ovipositor sheath approximately 1.1 times that of metasoma, and about 0.7 times length of forewing. Setosity of ovipositor sheath dense and evenly short.

Head brown. Mesosoma reddish-brown. Antenna and ovipositor sheath dark brown. Legs and palpi pale yellow. Metasoma sub-laterally and 4th-6th tergites brown, 2nd-3rd tergites medially dark brown.

Type material. Holotype, $q$ (China): "Zhejiang, Jinyun, Li Si-chun", collection date unknown.

Etymology. From "grandis" and "venter", Latin for large abdomen, referring to the shape of the metasoma.

Distribution. China (Zhejiang).

\section{Testudobracon pleuralis (Ashmead, 1906)}

(Figs 28-31)

Chelonogastra pleuralis Ashmead, 1906: 196; Watanabe, 1934: 184.

Iphiaulax (Chelonogastra) pleuralis; Fahringer, 1928: 591.

Philomacroploea pleuralis; Watanabe, 1937: 17; Shenefelt, 1978: 1714; Achterberg, 1984: 151.

Testudobracon pleuralis; Tang et al., 2001: 152.

Material examined. 19 (China): "Hubei, Tongcheng, 6.vii. 1978, Luo Qi- gui". 1ㅇ (China): "Zhejiang, Hangzhou, 12.vii.1979, Lui Shao-hua".

Distribution. China (Hubei, Zhejiang, Taiwan), Japan.
Biology. Asphondylia sp. (Diptera, Cecidomyiidae) on Glycine max (Linn.) Merr (the soybean pod gall midge). The host record Etiella zinckenella (Treitschke, 1832) (Lepidoptera: Pyralidae) needs confirmation (Maetô, 1991; Tang et al., 2001).

ACKNOWLEDGEMENTS. We wish to thank Donald L.J. Quicke (London, U.K.) for his critical review of the first draft of this paper. The project was supported by a grant from the National Natural Science Foundation of China (NSFC Number: 39970099) to the second author.

\section{REFERENCES}

Achterberg van C. 1979: A revision of the Subfamily Zelinae auct. (Hymenoptera, Braconidae). Tijdschr. Entomol. 122: 241-479.

ACHTERBERG VAN C. 1984: Revision of the genera of Braconini with first and second metasomal tergites immovably joined (Hymenoptera: Braconidae, Braconinae). Tijdschr. Entomol. 127: $137-164$.

Ashmead W.H. 1906: Descriptions of new Hymenoptera from Japan. Proc. U. S. Nat. Mus. 30: 169-201.

FAHringer J. 1928: Opuscula Braconologica. Palaearktische Region. Lief. 1. F. Wagner, Wien, pp. 433-606.

MAETô K. 1991: Braconid parasitoids (Hymenoptera) of the gall-making Cecidomyiidae (Diptera) in Japan. Jap. J. Entomol. 59: 295-313.

Quicke D.L.J. 1986: Seven new genera and species of Braconinae (Hymenoptera, Braconidae) from Australasia and Indonesia. Entomol. Mon. Mag. 122: 9-29.

QUicke D.L.J. 1987: The old world genera of braconine wasps (Hymenoptera: Braconidae). J. Nat. Hist. 21: 43-157.

QUiCKE D.L.J. \& INGRAM S.N. 1993: Braconine wasps of Australia. Mem. Queensl. Mus. 33: 299-336.

Shenefelt R.D. 1978: Hymenoptera Catalogus (Nov. Ed.). Part 15. Braconidae, 10. W. Junk, The Hague, pp. 1425-1865

Tang Jian, Belokobylski S.A. \& Chen Xue-Xin 2001: New records of braconid parasitoids (Hymenoptera) from paddy field in China. Entomotaxon. 23: 152.

Watanabe C. 1934: H. SAUTER'S-Collection: Braconidae. Insect. Mats. 8: 182-205.

Watanabe C. 1937: A contribution to the knowledge of the braconid of the Empire of Japan. J. Fac. Hokk. Imp. Univ. 42: $1-188$.

Received July 18, 2002; revised October 10, 2002; accepted December 9, 2002 